\title{
Response to Letter to the Editor: "Safety profile of intravenous patient-controlled analgesia for breakthrough pain in cancer patients: a case series study"
}

\author{
A. M. Sousa • G. M. N. Guimaraes • H. A. Ashmawi
}

Received: 30 June 2014 / Accepted: 31 July 2014 /Published online: 9 August 2014

(C) Springer-Verlag Berlin Heidelberg 2014

We thank Dr. Davies for the interest in our work and for his comments.

About the specific comment on the title of the paper, it is difficult to assess breakthrough pain (BTP), especially in patients with incident pain who avoid certain movements that might trigger pain. The criteria to include patient-controlled analgesia (PCA) in the present work were "if a certain patient presented with severe BTP requiring at least five doses or rescue systemic opioid analgesia or previous attempt to improve pain control." In the next paragraph, we used the definition described by Haugen et al. [1], that can certainly be misunderstood in the present reading. The questions pointed by Dr. Davies concerning the review article by Haugen et al. [1] are correct, "it's certainly questionable to characterize and treat BTP when baseline pain is not controlled." We agree with this.

However, we described the use of PCA in patients with and without BTP. All patients described in this work had been submitted to the standard therapy provided by the oncologist; the most difficult to treat had "incident pain" or "movementrelated pain," but were pain-free most of the time. Some patients, that we diagnosed as suffering from BTP, were hospitalized and had to leave their rooms several times a day, in order to be submitted to procedures, and suffered pain when moved from one place to the other. That type of pain is in accordance to the definition of BTP described by Davies [2].

As to the observation about other therapeutics options, in our institution, PCA is a safer, timely, and useful system than traditional methods to treat patients with severe BTP but is certainly not the only one.

In conclusion, we are thankful for the comments and for pointing out further research possibilities.

Conflict of interest The authors declare no conflicts of interest.

\section{References}

1. Haugen DF, Hjermstad MJ, Hagen N, Caraceni A, Kaasa S (2010) On behalf of the European Palliative Care Research Collaborative (EPCRC). Assessment and classification of cancer breakthrough pain: a systematic literature review. Pain 149:476-482

2. Davies AN (2014) Curr Pain Headache Rep 18:420
A. M. Sousa $(\varangle) \cdot$ G. M. N. Guimaraes

Cancer Institute of the State of Sao Paulo, Sao Paulo, Brazil

e-mail: angela-sousa@uol.com.br

H. A. Ashmawi

Hospital das Clinicas, University of Sao Paulo, Sao Paulo, Brazil 